\title{
Is place the (only) functional correlate?
}

\author{
H. EICHENBAUM and S. I. WIENER \\ Wellesley College, Wellesley, Massachusetts
}

\begin{abstract}
To assess whether "place" is necessary and sufficient to account for the functional correlates of hippocampal neuronal activity, we examined several spatial and behavioral parameters associated with unit activity in rats performing both a place-guided navigation task and an odorguided sensory-discrimination task. In the place task, most cells had place fields, but were also activated selectively in relation to speed, direction, or turning angle of movements the rat made in the field. In the odor task, most cells fired selectively either during the period of odor sampling and response generation or as the rat initiated the trial or approached the reward. Some of these cells fired preferentially in association with the presentation of particular stimulus configurations. Often the same cells had clear, but unrelated, spatial correlates in the place task and behavioral correlates in the odor task. These results suggest that we must view spatial coding as only one form of cognitive processing performed by the hippocampus, and that both spatial and behavioral variables must be considered in a complete account of hippocampal neuronal activity.
\end{abstract}

Other papers presented in this workshop have provided strong confirmation of the participation of hippocampal complex spike cells in spatial processing and have helped to illuminate some of the characteristics of place coding. However, we have only begun to address the issue of whether place is the only-or even the primaryfunctional correlate of hippocampal neuronal activity.

As we consider what the place-field phenomenon tells us about hippocampal function, we must keep in mind that spatial mapping and spatial memory impairment play no special role in the global amnesia resulting from hippocampal system damage in humans, suggesting to many that the hippocampus serves a more general memory function. Consistent with this view, we begin with a reminder that several investigators have described nonspatial correlates of hippocampal unit activity, some of which are at least as impressive as place fields. For example, Berger, Thompson, and colleagues (e.g., Berger, Rinaldi, Weisz, \& Thompson, 1983) have demonstrated a nonspatial behavioral correlate of CA1 pyramidal cell activity wherein increases in neuronal activity are time-locked to the conditioned and unconditioned stimuli in Gormezzano's paradigm for classical conditioning of the eyeblink. This sort of finding raises a question central to today's workshop: Is it "place" that hippocampal neurons actually code? To address this issue, we sought to determine whether the animal's location in space is sufficient to explain the variation in cell firing rate in spatial paradigms, and whether place is a necessary variable in accounting for hippocampal complex spike cell activity in nonspatial conditioning tasks.

This work was supported by the PHS (NS26402) and NSF (BNS 8810095). A more detailed account of the methods and results of this report are given in Wiener, Paul, and Eichenbaum (in press). Address correspondence to Howard Eichenbaum, Department of Biological Sciences, Wellesley College, Wellesley, MA 02181.
We evaluated the functional correlates of CA1 neurons recorded in rats that sequentially performed two tasksone a spatial navigation task and the other a spaceirrelevant, sensory-discrimination task using odor cues that depend on hippocampal function (Eichenbaum, $\mathrm{Fa}$ gan, Mathews, \& Cohen, 1988). Both tasks were performed in the same environment, a $40-\mathrm{cm}^{2}$ arena with a cul-de-sac on one wall (Figure 1). The place task required rats to move back and forth from the center of the arena to each corner, where a water reward was given, and to remember locations previously visited, because repeat visits to a corner within a trial were not rewarded. The odor-discrimination task required rats to await an auditory signal, then enter the cul-de-sac in order to turn on odor cues that were emitted from two stimulus ports. They then performed a nosepoke into the port associated with a particular odor to receive a water reward.

We asked three questions about the functional correlates of neuronal activity in these tasks. First, to what extent is ongoing behavior an important consideration in the spatial correlates of unit activity observed during performance of the place task? Second, to what extent do spatial factors account for behavioral correlates seen in the odor task? And third, do the same cells have both place fields in the place task and behavioral correlates in the odor task, and if so, are these correlates related?

We analyzed spatial firing by dividing the environment into a matrix of pixels and evaluating the reliability of increased unit activity upon repeated visits to each pixel. Figure 2 shows an example of our analyses on more than 400 cells in the place task. This cell had a relatively large place field between the center of the arena and Cup 3 . But its firing rate was also dependent on the rat's actions within the field, as seen in the associated histograms and polar plots. First, the cell fired preferentially when the rat was moving at intermediate to high speeds, rather than 


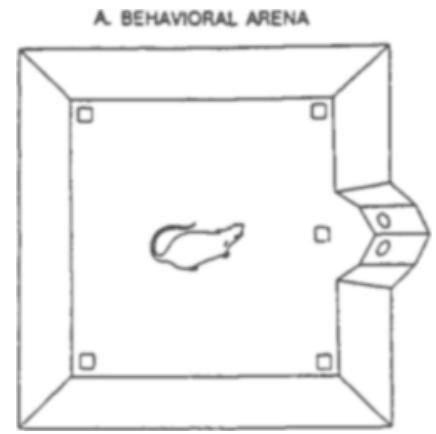

B. PLACE TASK

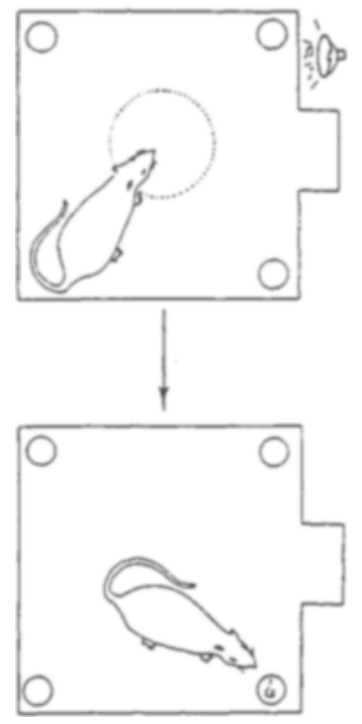

Figure 1. The behavioral apparatus (A), and place-task and odortask paradigms. (B) In the place task, rats must reach the center of the arena to initiate a run (upper panel), then approach a cup not previously visited on that trial (lower panel). (C) In the odor task, rats enter the cul-de-sac to initiate odor onset (upper panel enlarged view of the cul-de-sac), then perform a nosepoke at the port where the $\mathrm{S}^{+}$is presented (lower panel).

slower speeds. Second, the cell fired preferentially when the rat was moving in the southerly direction. And third, the cell fired preferentially when the rat was turning to the left. In one more analysis, we also time-locked unit activity to the approach movement associated with reward delivery, separately for the approach to each of the four corners of the arena. This cell's activity was time-locked just prior to the animal's arrival at Cup 3. Of course, the time-locked firing of this cell can be explained in terms of when the rat would be expected to be in the place field, but it is also the case that the place field can be explained by the combination of movement-related and time-locked firing characteristics. Our conclusion is that both the place and the behavioral correlates contribute significantly to a full accounting of cellular activity, and that ignoring either place or behavioral variables would result in an incomplete description of the functional correlate.
The analyses of many other examples reinforced this interpretation. In particular, some cells had a place field composed of multiple subfields, and within each subfield there was a distinct movement-correlate profile. Most striking perhaps was that some of these cells fired maximally as the rat moved in opposite directions within each subfield. Combining the place and movement correlates of these cells, their activity could be described best in terms of maximal firing when the rats were at multiple locations making specific movements toward specific spatially defined goals.

Using the odor task, we also asked to what extent place and behavior are important in accounting for unit activity during sensory-discrimination learning. Our analyses of more than 250 hippocampal units showed that in about half of the cells, increased firing was associated with specific behavioral events. The raster display and perievent histogram for the analyses of one cell shown in Figure 3A led us to conclude that this cell fired after the onset of odor presentation, indicated by tic marks for individual trials, and prior to the discriminative response, indicated by the aligned tic marks at the center of the figure. Because firing was selective to the period when the rat was sampling the odors and generating a response, we refer to these cells as "cue-sampling cells" (Eichenbaum, Kuperstein, Fagan, \& Nagode, 1987). In addition, the firing of many of these cells was selective to particular odors presented in a particular configuration (including the example in Figure 3; see below).

To investigate the importance of spatial factors in unit activity recorded as the rats performed the odor task, we superimposed on an outline of the cul-de-sac, with dots indicating the successive locations of the rat's snout at 50 msec intervals and squares indicating unit firing associated with those loci for each of three 1 -sec periods over several trials (Figure 3B). Our analysis of the cell shown in Figure $3 \mathrm{~A}$ indicated that firing was spatially localized, but that place alone was a poor predictor of unit activity. The cell fired only when the rat was just in front of either of the odor ports, but firing occurred only during the cuesampling period (Figure 3B, left) and not during the 1sec period following nosepoke onset (Figure 3B, right), even though the rat was in the same place facing the same direction. We realize, of course, that the rat's behavior, including movement direction, was different in these two trial phases, but that is just the point. This is a behavioral correlate that happens to occur at particular places.

Other cells had behavioral correlates that were complementary to that of cue-sampling cells. These cells, called "goal-approach cells," typically did not fire during the cue-sampling period, but burst after the rat completed the discriminative response, while it was still at the odor port facing in the same direction and beginning to move toward the reward. Other goal-approach cells fired selectively during the initial approach to the sampling ports as the trial began. In many cases, the firing of these cells was not spatially localized. 

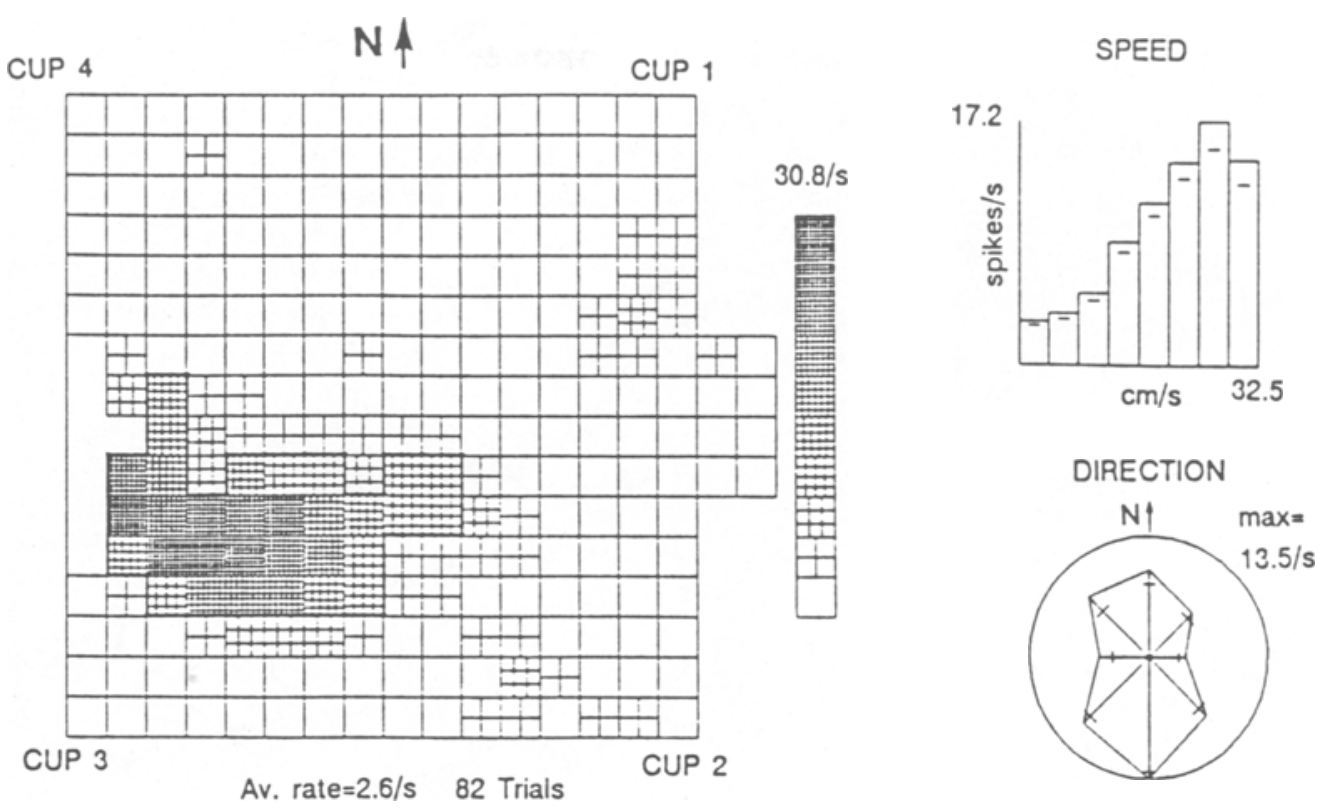

TIME-LOCK TO CUP ARRIVAL

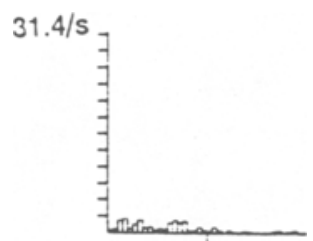

CUP 1

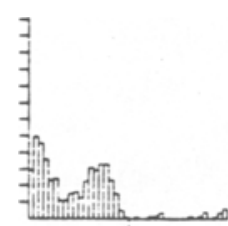

CUP 2

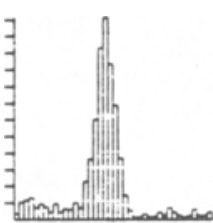

CUP 3

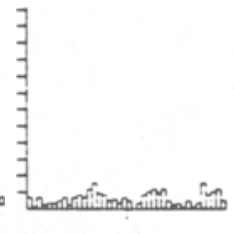

CUP 4

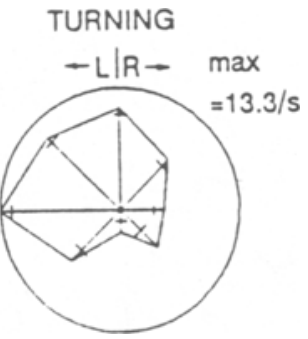

Figure 2. Analyses of spatial and behavioral correlates of a hippocampal cell in a rat performing the place task. Panels include a map of firing rates throughout the arena, with the place field outlined (top left), a histogram and polar plots showing tuning to movement parameters (right), and histograms of firing rate time-locked at the center of each plot (4-sec total period) to the arrival at each water cup (bottom left).

It behooves us to ask whether space plays any significant role in the behavioral correlates observed in this task. The answer is that several cue-sampling and goal-approach cells fire preferentially according to the odors presented, the positions of the odors and response, or both. Firing in relation to odor positions was assessed by having the rats perform two odor-discrimination problems concurrently on separate trials. We distinguished the unit activity associated with different odor pairs presented in different left-right configurations. The activity of several cells was preferentially increased in association with the conjunction of a particular odor and position. Thus, space does sometimes play a critical role in the behavioral correlates of hippocampal neuronal activity during the performance of sensory discriminations. To be more specific, the positions of cues and responses seem to be encoded in hippocampal activity. This spatial aspect of the behavioral correlate is, of course, not that of a Euclidean spatial map; it is more simply described by the spatial configuration of the odor cues and responses.

Finally, we asked whether the same cells that have spatial correlates in the place task also have behavioral corre- lates in the odor task. In brief, clear correlates of both types are seen in some cells, but the relationship between the two correlates is unclear. Figure $3 \mathrm{C}$ shows the analyses of a cell that acted as a cue-sampling cell in the odor task (Figures $3 \mathrm{~A}$ and $3 \mathrm{~B}$ ). In the place task, this cell acted as a place cell with a single, clear place field distant from the cul-de-sac, and was selectively tuned for movement speed, direction, and turning. (Lest we conclude that this cell is poorly tuned to speed, note the speed curve plotted for movements only in the optimal direction and turning angle.)

Since both tasks were performed in the same environment, a strict spatial mapping hypothesis would predict that, in the place task, cells with behavioral correlates in the odor task would have place fields located at the culde-sac. However, this was not so. The place fields of cells that had behavioral correlates in the odor task, and those of cells that had no clear behavioral correlate, were distributed more or less evenly throughout the environment.

In summary, we found that (1) the activity of hippocampal cells in a place task is dependent on behavior in space, 

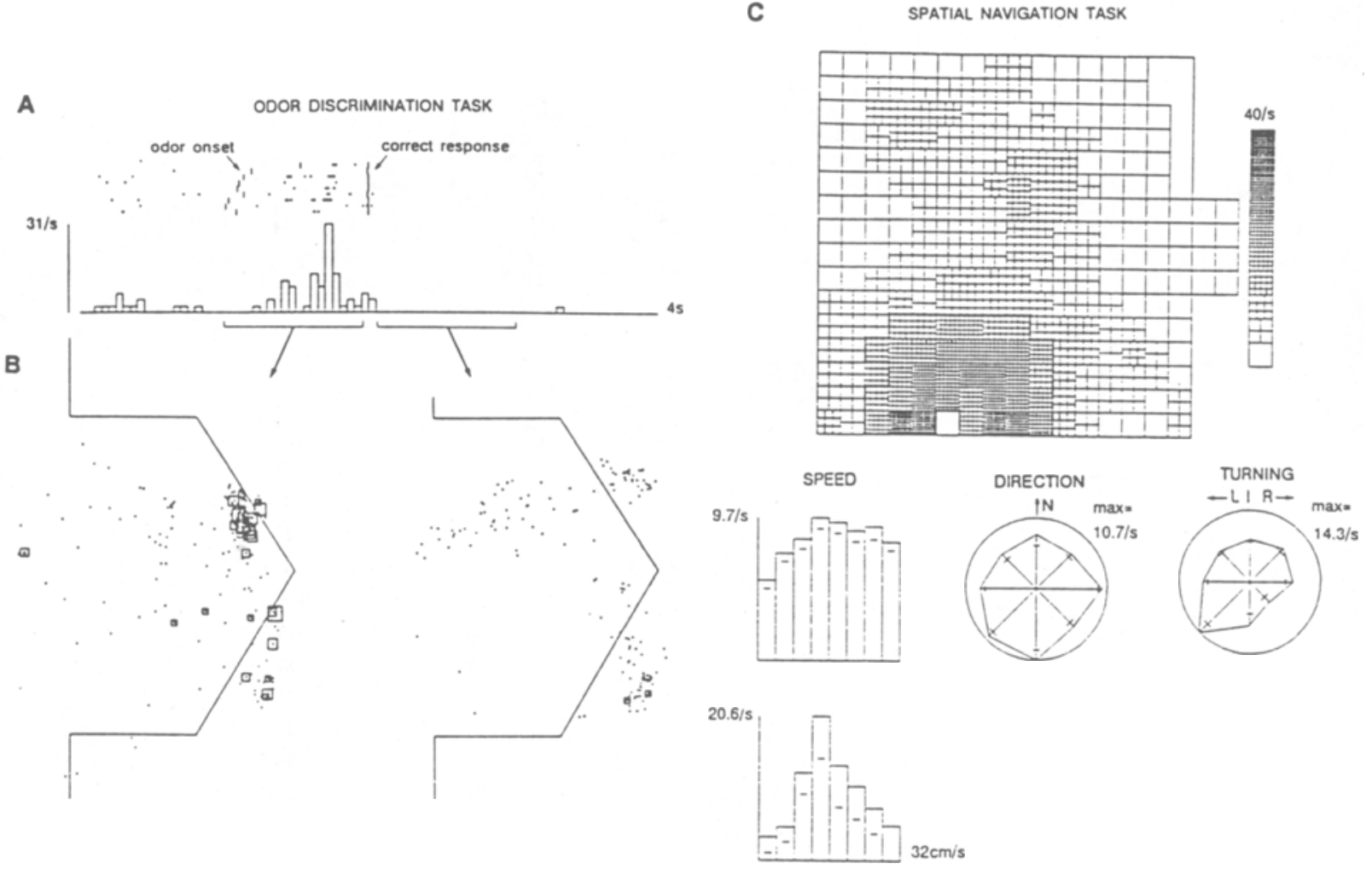

Figure 3. Analyses of spatial and behavioral correlates of a cell recorded in a rat performing the odor task (Panels A and B) and performing the place task (Panel C). (A) Raster display and summary histogram of firing time-locked to the discriminative response during presentation of a particular odor configuration. (B) Enlarged schematic view of the cul-de-sac plotting location of the rat (dots) and firing rate (squares) at 50-msec intervals for 1-sec periods indicated in Panel $A$. (C) The cell had a distinct place field and was significantly tuned to speed $\left[\chi^{2}(7)=49.1, p<.001\right]$, direction $\left[\chi^{2}(7)=29.4, p<.001\right]$, and turning angle $\left[\chi^{2}(7)=30.0, p<.001\right]$. When the speed-tuning curve was plotted only for preferred movement direction (bottom), speed tuning was also significant $\left[\chi^{2}(7)=22.2, p<.01\right]$.

not on place alone; (2) in the odor task, space plays at best a secondary role in the behavioral correlates of hippocampal unit activity; and (3) the same cells can encode either primarily spatial or primarily nonspatial variables in different tasks performed in the same environment. These findings suggest that although aspects of space powerfully engage hippocampal processing, spatial mapping per se is neither sufficient nor necessary to account for hippocampal processing. In conclusion, we seek a less restrictive view of hippocampal function, one that can explain the global amnesia observed in humans (Eichenbaum \& Cohen, 1988). Furthermore, the present data tell us that a complete account of hippocampal neuronal activity must incorporate the processing of both spatial and nonspatial stimulus relationships and, even in spatial tasks, must incorporate actions toward and choices among stimuli.

\section{REFERENCES}

Berger, T. W., Rinaldi, P. C., Weisz, D. J., \&hompson, R. F. (1983). Single-unit analysis of different hippocampal cell types during classical conditioning of the nictitating membrane response. Joumal of Neurophysiology, 50, 1197-1219.

Eichendaum, H., Cohen, N. J. (1988). Representation in the hippocampus: What do hippocampal neurons code? Trends in Neuroscience, 11, 244-248.

Eichendaum, H., Fagan, A., Mathews, P., Cohen, N. J. (1988). Hippocampal system dysfunction and odor discrimination learning in rats: Impairment or facilitation depending on representational demands. Behavioral Neuroscience, 102, 331-339.

Eichendaum, H., Kuperstein, M., FAgan, A., \& Nagode, J. (1987). Cue-sampling and goal-approach correlates of hippocampal unit activity in rats performing an odor discrimination task. Journal of Neuroscience, 7, 716-732.

Wiener, S. I., Paul, C. A., \&ichenbaum, H. (in press). Spatial and behavioral correlates of hippocampal neuronal activity. Journal of Neuroscience. 